\title{
SOME TYPICAL PROPERTIES OF DIMENSIONS OF SETS AND MEASURES
}

\author{
JÓZEF MYJAK
}

Received 2 January 2004

This paper contains a review of recent results concerning typical properties of dimensions of sets and dimensions of measures. In particular, we are interested in the Hausdorff dimension, box dimension, and packing dimension of sets and in the Hausdorff dimension, box dimension, correlation dimension, concentration dimension, and local dimension of measures.

\section{Introduction}

The idea of dimension of sets and measures is fundamental in diverse branches of mathematics, among other in the theory of measure, in the theory of dynamical system and in the theory of fractals. Various notions of dimension have been proposed: Hausdorff dimension (undoubtedly the most popular), box dimension, correlation dimension, packing dimension, informatic dimension, entropy, and capacity. All these concepts were widely investigated and used and all of them are rather hard to calculate.

The study of typical properties of compact sets has had a long story and ample literature. Many of related results can be found in survey papers by Gruber [9] and Zamfirescu $[34,35,36]$. Here we present some recent results concerning typical properties of dimensions of sets and dimensions of measures.

Recall that a subset of a metric space $X$ is called of the first Baire category, if it can be represented as a countable union of nowhere dense sets. A subset of a complete metric space is called residual, if its complement is of the first Baire category. If the set of all elements of $X$ satisfying some property $P$ is residual in $X$, then property $P$ is called generic or typical. We say that an element of $X$ is typical if it has property $P$.

To recall some classical observations concerning typical properties of dimensions of sets, we assume that the space of compact sets is equipped with the Hausdorff distance. From [28] it follows that a typical compact subset of a separable metric space $X$ has Hausdorff dimension zero. Since the covering (Lebesgue) dimension is less than or equal to the Hausdorff dimension, this implies that a typical compact set is totally disconnected. Moreover, if the space $X$ is connected, a typical compact set has no isolated points. In [10], Gruber proved that if the collection of compact sets having lower box dimension 
at least $\delta$ is dense in the space of compact sets, then a typical compact set has upper box dimension at least $\delta$. In [6], Feng and Wu proved that a typical compact set in $\mathbb{R}^{n}$ has packing dimension $n$. On the other hand, Haase [11] proved that, given $x \in X$, for a typical measure $\mu$, the lower local dimension at $x$ is equal to zero. In addition, if $X$ has no isolated points, then upper local dimension at $x$ is equal to infinity. This result was generalized by Genyuk [8] by proving that for a typical probability measure $\mu$ on a Polish space $X$, the lower local dimension of $\mu$ is equal to zero and the upper local dimension of $\mu$ is equal to $\infty$, for all $x$ except a set of the first category.

The study of typical properties of Markov (stochastic) operators is the other related field of research. The reader is referred to the review article by Choksi and Prasad [2] for information on historic background and significance of Baire category theorems in ergodic theory. For example, it was shown that a typical stochastic operator is conservative and ergodic for the strong topology [15]. Moreover, a typical stochastic operator acting on arbitrary $\sigma$-finite measure space is completely mixing for both strong and uniform topologies (see $[1,31])$.

In [16], it was proved that a typical stochastic semigroup generated by iterated function system is asymptotically stable and has a strictly positive invariant density. This result was generalized in [31] by proving that the set of convergent functions is residual in the space of all multiplicative functions. In [17], it was shown that a unique stationary measure corresponding to an iterated function system is either absolutely continuous or singular. Moreover, in the family of all nonexpansive iterated function system the last property is generic. This result was generalized in [33] by proving that a typical iterated function system with place dependent probabilities is asymptotically stable and nonexpansive. Finally, in [24], it was proved that a typical Markov operator acting on the space of Borel measures supported on a compact subset of $\mathbb{R}^{d}$ is asymptotically stable and has singular stationary measure.

Although first results concerning typical properties of dimensions appeared also a long time ago, on the contrary to other geometric properties of sets and measures, generic properties of dimensions does not seem to be so extensively studied. Some results in this direction have been appeared quite recently and here we want to give a review of these results. As a general rule, we will give only the frame of the proofs. Complete proofs can be found in $[8,11,18,20,21,22,23,24,25,26]$.

\section{Preliminaries}

Let $(X, \rho)$ be a complete metric space. By $B(x, r)$ (resp., $\left.B^{o}(x, r)\right)$ we denote the closed (resp., open) ball in $X$ with centre at $x$ and radius $r>0$. For $A \subset X, \operatorname{cl} A$ stands for the closure of $A, \operatorname{diam} A$ for the diameter of $A$, and $\operatorname{card} A$ for the cardinality of $A$. As usual, by $\mathbb{R}$ and $\mathbb{N}$ we denote the sets of all reals and all positive integers, respectively.

By $\mathcal{K}$ we denote the family of all nonempty compact subsets of $X$ endowed with the Hausdorff distance $h$ given by the formula

$$
h(A, B)=\max \left\{\max _{x \in A} \rho(x, B), \max _{x \in B} \rho(x, A)\right\}, \quad A, B \in \mathscr{K},
$$


where

$$
\rho(x, B)=\min \{\rho(x, y): y \in B\} .
$$

It is well known that the metric space $(\mathscr{K}, h)$ is complete.

For $A \subset X, s \geq 0$ and $\delta>0$, define

$$
\mathscr{H}_{\delta}^{s}(A)=\inf \sum_{i=1}^{\infty}\left(\operatorname{diam} U_{i}\right)^{s},
$$

where the infimum is taken over all countable covers $\left\{U_{i}\right\}$ of $A$ such that $0<\operatorname{diam} U_{i} \leq \delta$. Then

$$
\mathscr{H}^{s}(A)=\lim _{\delta \rightarrow 0} \mathscr{H}_{\delta}^{s}(A)
$$

is called the Hausdorff s-dimensional measure. If $\mathscr{H}^{s}(A)<\infty$ for some $s>0$, we define the Hausdorff dimension of $A$ by the formula

$$
\operatorname{dim}_{H} A=\inf \left\{s>0: \mathscr{H e}^{s}(A)<\infty\right\} .
$$

If $\mathscr{H}^{s}(A)=\infty$ for every $s>0$, we set $\operatorname{dim}_{H} A=\infty$.

By $\mathscr{B}(X)$ we denote the $\sigma$-algebra of Borel subsets of $X$ and by $\mu_{1}(X)$ the family of all probability Borel measures on $X$, that is, the measures $\mu$ on $\mathscr{B}(X)$ such that $\mu(X)=1$.

The Hausdorff dimension of a measure $\mu \in M_{1}(X)$ is defined by the formula

$$
\operatorname{dim}_{H} \mu=\inf \left\{\operatorname{dim}_{H} A: A \in \mathscr{B}(X), \mu(A)=1\right\} .
$$

Given $\mu \in \mathcal{M}_{1}(X)$, we define the support of $\mu$ by the formula

$$
\operatorname{supp} \mu=\{x \in X: \mu(B(x, r))>0 \text { for every } r>0\} .
$$

As usual, by $B(X)$ we denote the space of all bounded Borel measurable functions $f$ : $X \rightarrow \mathbb{R}$ and by $C(X)$ the subspace of all continuous functions. Both spaces are considered with the supremum norm.

For $f \in B(X)$ and $\mu \in \mathcal{M}_{1}(X)$, we write

$$
\langle f, \mu\rangle=\int_{X} f(x) \mu(d x) .
$$

We admit that the space $M_{1}(X)$ is endowed with the Fortet-Mourier distance $d_{\mathrm{FM}}$ defined by the formula

$$
d_{\mathrm{FM}}(\mu, \nu)=\sup \{|\langle f, \mu\rangle-\langle f, \nu\rangle|: f \in \mathscr{L}\},
$$

where $\mathscr{L}$ is the set of all $f \in C(X)$ such that $|f(x)| \leq 1$ and $|f(x)-f(y)| \leq \rho(x, y)$ for $x, y \in X$ (see [7]). 
We say that a sequence $\left(\mu_{n}\right) \subset M_{1}(X)$ converges weakly to a measure $\mu \in M_{1}(X)$ if

$$
\lim _{n \rightarrow \infty}\left\langle f, \mu_{n}\right\rangle=\langle f, \mu\rangle \quad \text { for every } f \in C(X) .
$$

It is well known (see [3]) that the convergence in the Fortet-Mourier metric is equivalent to the weak convergence. Moreover, $\left(M_{1}(X), d_{\mathrm{FM}}\right)$ is a complete metric space.

\section{Dimensions of compact sets}

We recall that the lower and upper box dimensions of a set $A \subset X$ are defined, respectively, by the formulae

$$
\begin{aligned}
& \underline{\operatorname{dim}}_{b} A=\liminf _{r \rightarrow 0^{+}} \frac{\log N(A, r)}{\log (1 / r)}, \\
& \overline{\operatorname{dim}}_{b} A=\limsup _{r \rightarrow 0^{+}} \frac{\log N(A, r)}{\log (1 / r)},
\end{aligned}
$$

where $N(A, r)$ is the least number of closed balls of radius $r$ which cover the set $A$. Note that if the closure of the set $A$ is noncompact, then $\overline{\operatorname{dim}}_{b} A=\underline{\operatorname{dim}}_{b} A=\infty$. Note also that the lower and upper box dimensions are invariant under the topological closure. Moreover, it is well known that the Hausdorff dimension of $A$ is not larger than the lower box dimension of $A$.

In the above definitions of box dimensions, we can replace the number $N(A, r)$ by $M(A, r)$ - the greatest possible number of disjoint closed balls of radius $r$ that may be found with centers in $A$.

Finally note that the box dimension defined above, eventually with some modifications, was intensively studied for a long time and it is known in literature also under the name Minkowki dimension, fractal dimension, capacity dimension, or entropy dimension. (See [5] and references therein.)

Theorem 3.1. A typical compact subset of $X$ has Hausdorff and lower box dimension zero.

Proof. Since the lower box dimension is greater than or equal to the Hausdorff dimension (see [12]), it suffices to consider only the case of lower box dimension. Denote by $\mathscr{F}$ the family of all finite subsets of $X$ and by $c^{+}$the family of all decreasing sequences of positive numbers. For $\mathbf{a}=\left(a_{1}, a_{2}, \ldots\right) \in c^{+}$, define

$$
\mathscr{B}(\mathbf{a})=\bigcup_{F \in \mathscr{F}} \mathscr{B}(F, \mathbf{a})
$$

where

$$
\mathscr{B}(F, \mathbf{a})=\left\{A \in \mathscr{K}: h(A, F)<a_{n}\right\}, \quad n=\operatorname{card} F .
$$

Now for a given sequence $\left(\mathbf{a}^{k}\right)$ of elements of $c^{+}$we set

$$
\mathscr{B}\left(\mathbf{a}^{1}, \mathbf{a}^{2}, \ldots\right)=\bigcap_{k=1}^{\infty} \mathscr{\mathscr { B }}\left(\mathbf{a}^{k}\right) .
$$

It is easy to verify that the set $\mathscr{B}\left(\mathbf{a}^{1}, \mathbf{a}^{2}, \ldots\right)$ is residual in $\mathscr{Y}$. 
We claim that for $A \in \mathscr{B}\left(\mathbf{a}^{1}, \mathbf{a}^{2}, \ldots\right)$, where $a_{n}^{k}=2^{-k-n}$, we have $\underline{\operatorname{dim}}_{b} A=0$. Indeed, by the definition of $\mathscr{B}\left(\mathbf{a}^{1}, \mathbf{a}^{2}, \ldots\right)$ and the choice of sequence $\left(\mathbf{a}^{k}\right)$, for every $k \in \mathbb{N}$, there exists a finite set $F=\left\{x_{1}, \ldots, x_{n(k)}\right\}$ such that

$$
h(A, F)<2^{-k-n(k)} .
$$

Set $\varepsilon_{k}=2^{-k-n(k)}$. Then $N\left(A, \varepsilon_{k}\right) \leq n(k)$ and consequently

$$
0 \leq \underline{\operatorname{dim}}_{b} A \leq \lim _{k \rightarrow \infty} \frac{\log _{2} N\left(A, \varepsilon_{k}\right)}{\log _{2}\left(1 / \varepsilon_{k}\right)} \leq \lim _{k \rightarrow \infty} \frac{\log _{2} n(k)}{k+n(k)}=0 .
$$

This completes the proof.

THeORem 3.2. If the family of compact sets having lower box dimension at least $\delta$ is dense in $\mathscr{K}$, then a typical compact set has upper box dimension at least $\delta$.

For the proof, see [10].

The smallest local lower and the smallest local upper box dimension of the set $A$ are defined, respectively, by formulae

$$
\begin{aligned}
& \text { sl- } \underline{\operatorname{dim}}_{b} A=\inf \left\{\underline{\operatorname{dim}}_{b}(B(x, r) \cap A): x \in A, r>0\right\}, \\
& \text { sl- } \overline{\operatorname{dim}}_{b} A=\inf \left\{\overline{\operatorname{dim}}_{b}(B(x, r) \cap A): x \in A, r>0\right\} .
\end{aligned}
$$

If $A=X$, we obtain the smallest local lower and the smallest local upper box dimension of the space $X$.

Using these concepts, we can generalize Theorem 3.2 in the following way.

Theorem 3.3. For a typical compact subset $A$ of $X$,

$$
\text { sl }-\overline{\operatorname{dim}}_{b} A \geq \mathrm{sl}-\overline{\operatorname{dim}}_{b} X .
$$

Proof. If sl $-\overline{\operatorname{dim}}_{b} X=0$, the statement is obvious. Assume that $\mathrm{sl}-\overline{\operatorname{dim}}_{b} X>\delta>0$. For $x \in$ $X, r>0$ and $\varepsilon>0$ by $M(x, r ; \varepsilon)$, we denote the greatest possible number of disjoint closed balls of radius $\varepsilon$ and with centers in the ball $B(x, r)$. Since $\overline{\operatorname{dim}}_{b} B(x, r)>\delta$, there exists $\varepsilon=\varepsilon(x, r, \delta)<1$ such that

$$
M(x, r ; \varepsilon) \geq \varepsilon^{-\delta}
$$

Clearly $\varepsilon(x, r, \delta)<r$. Let $D(x, r, \varepsilon)$ be a subset of $B(x, r)$ having $M(x, r, \varepsilon)$ elements and such that $\rho\left(y_{1}, y_{2}\right)>2 \varepsilon$ for every $y_{1}, y_{2} \in D(x, r, \varepsilon), y_{1} \neq y_{2}$.

Denote by $\mathscr{F}$ the family of all finite subsets of $X$. For $F \in \mathscr{F}$ and $n \in \mathbb{N}$, we define

$$
\mathscr{B}_{n}(F, \delta)=\left\{A \in \mathscr{K}: h\left(A, \bigcup_{x \in F} D\left(x, \frac{1}{n}, \varepsilon\right)\right)<\alpha\left(F, \frac{1}{n}, \delta\right)\right\},
$$

where

$$
\alpha\left(F, \frac{1}{n}, \delta\right)=\frac{1}{3} \min _{x \in F} \varepsilon\left(x, \frac{1}{n}, \delta\right) .
$$


Let

$$
\mathscr{B}(\delta)=\bigcap_{m=1}^{\infty} \bigcup_{n=m}^{\infty} \mathscr{B}_{n}(\delta) \text {, where } \mathscr{P}_{n}(\delta)=\bigcup_{F \in \mathscr{F}} \mathscr{B}_{n}(F, \delta) .
$$

It is easy to see that the set $\mathscr{B}(\delta)$ is residual in the space $\mathscr{K}$.

We claim that

$$
\text { sl }-\overline{\operatorname{dim}}_{b} A \geq \delta \quad \text { for } A \in \mathscr{B}(\delta)
$$

Indeed, let $A \in \mathscr{B}(\delta), x_{0} \in A$, and $r>0$. Clearly, there exist an increasing sequence $\left\{n_{k}\right\} \subset \mathbb{N}$ and a sequence $\left\{F_{k}\right\} \subset \mathscr{F}$ such that

$$
h\left(A, \bigcup_{x \in F_{k}} D\left(x, \frac{1}{n_{k}}, \varepsilon\right)\right)<\alpha\left(F_{k}, \frac{1}{n_{k}}, \delta\right), \quad k=1,2, \ldots
$$

From (3.14) and the definition of $\varepsilon$, it follows that for each $k \geq 1$ there exists a point $x_{k} \in F_{k}$ such that $\rho\left(x_{0}, x_{k}\right)<2 / n_{k}$. Let

$$
\begin{gathered}
\alpha_{k}=\alpha\left(F_{k}, \frac{1}{n_{k}}, \delta\right), \quad \varepsilon_{k}=\varepsilon\left(x_{k}, \frac{1}{n_{k}}, \delta\right), \\
A_{k}=B\left(x_{0}, r\right) \cap\left\{y \in X: \rho(y, A) \leq \alpha_{k}\right\} .
\end{gathered}
$$

By (3.14) and the definition of $\alpha_{k}$, we have

$$
D\left(x_{k}, \frac{1}{n_{k}}, \varepsilon_{k}\right) \subset B\left(x_{0}, r\right) \quad \text { for } n_{k} \geq \frac{3}{r} .
$$

Consequently

$$
D\left(x_{k}, \frac{1}{n_{k}}, \varepsilon_{k}\right) \subset A_{k}
$$

and so

$$
M\left(A_{k}, \varepsilon_{k}\right) \geq M\left(D\left(x_{k}, \frac{1}{n_{k}}, \varepsilon_{k}\right), \varepsilon_{k}\right)=M\left(x_{k}, \frac{1}{n_{k}}, \varepsilon_{k}\right) \geq \varepsilon_{k}^{-\delta} .
$$

Let $U=A \cap B\left(x_{0}, r\right)$. Since for each point $y \in A_{k}$ there exists a point $y^{\prime} \in U$ such that $\rho\left(y, y^{\prime}\right) \leq \alpha_{k} \leq(1 / 3) \varepsilon_{k}$, we have

$$
M\left(U, \frac{\varepsilon_{k}}{3}\right) \geq M\left(A_{k}, \varepsilon_{k}\right) \geq \varepsilon_{k}^{-\delta}
$$

Consequently

$$
\overline{\operatorname{dim}}_{b} U \geq \limsup _{k \rightarrow \infty} \frac{\log M\left(U, \varepsilon_{k} / 3\right)}{-\log \left(\varepsilon_{k} / 3\right)} \geq \lim _{k \rightarrow \infty} \frac{\log \left(\varepsilon_{k}^{-\delta}\right)}{-\log \left(\varepsilon_{k} / 3\right)}=\delta,
$$

whence (3.13) follows. 
Now let $\left(\delta_{n}\right)$ be an increasing sequence of positive numbers convergent to sl- $\overline{\operatorname{dim}}_{b} X$. Set

$$
\mathscr{B}=\bigcap_{n=1}^{\infty} \mathscr{\mathscr { B }}\left(\delta_{n}\right)
$$

Clearly $\mathscr{B}$ is residual in $\mathscr{K}$. Let $A \in \mathscr{B}$. By (3.13) for arbitrary $n \in \mathbb{N}$, we have

$$
\text { sl }-\overline{\operatorname{dim}}_{b} A \geq \delta_{n},
$$

whence (3.8) follows.

Now using Theorem 3.3, we can estimate the packing dimension of the typical set. Recall that the packing dimension of a set $A \subset X$, (see $[3,26])$ can be defined by the formula

$$
\operatorname{dim}_{\mathrm{P}} A=\inf \left\{\sup _{1 \leq i<\infty} \overline{\operatorname{dim}}_{b} A_{i}: A=\bigcup_{i=1}^{\infty} A_{i}\right\} .
$$

It is clear that $\operatorname{dim}_{P} A \geq \overline{\operatorname{dim}}_{b} A$.

If $A$ is a closed set, we can assume that the sets $A_{i}$ in (3.23) are closed. Since the space $(A, \rho)$ has a Baire property, at least one of the sets $A_{i}$ has nonempty interior. Thus there exist $x \in A$ and $r>0$ such that $A \cap B(x, r) \subset A_{i}$. This implies that

$$
\operatorname{dim}_{P} A \geq \operatorname{sl}-\overline{\operatorname{dim}}_{b} A
$$

From the last inequality and Theorem 3.3, follows immediately the following corollary. Corollary 3.4. For a typical compact subset $A$ of $X$,

$$
\operatorname{dim}_{P} A \geq s \mathrm{~s}-\overline{\operatorname{dim}}_{b} X
$$

\section{Box dimensions of measures}

Let $\mu \in M_{1}(X)$. The quantities

$$
\begin{aligned}
& \underline{\operatorname{dim}}_{b} \mu=\lim _{\kappa \rightarrow 0^{+}} \inf \left\{\underline{\operatorname{dim}}_{b} A: A \in \mathscr{B}(X), \mu(A) \geq 1-\kappa\right\}, \\
& \overline{\operatorname{dim}}_{b} \mu=\lim _{\kappa \rightarrow 0^{+}} \inf \left\{\overline{\operatorname{dim}}_{b} A: A \in \mathscr{B}(X), \mu(A) \geq 1-\kappa\right\}
\end{aligned}
$$

are called the lower and the upper box dimension of $\mu$, respectively.

Theorem 4.1. For a typical measure $\mu \in M_{1}(X)$,

$$
\underline{\operatorname{dim}}_{b} \mu=0
$$


Proof. Denote by $\mathcal{N}$ the set of all measures $\mu \in M_{1}(X)$ such that cardinality of $\operatorname{supp} \mu$ is finite. For $v \in \mathcal{N}$, we define

$$
\mathscr{D}_{n}(\nu)=\left\{\mu \in M_{1}(X): d_{\mathrm{FM}}(\mu, \nu)<3^{-k-n}\right\},
$$

where $k=\operatorname{card}(\operatorname{supp} v)$.

Let

$$
\mathscr{D}=\bigcap_{n=1}^{\infty} \mathscr{D}_{n}, \quad \text { where } \mathscr{D}_{n}=\bigcup_{\nu \in \mathcal{N}} \mathscr{D}_{n}(\nu) \text {. }
$$

Since for each $n \in \mathbb{N}$ the set $\mathscr{D}_{n}$ is open and dense in the space $M_{1}(X)$, the set $\mathscr{D}$ is residual. To complete the proof it is sufficient to show that $\underline{\operatorname{dim}}_{b} \mu=0$ for every $\mu \in \mathscr{D}$.

Theorem 4.2. For a typical measure $\mu \in M_{1}(X)$,

$$
\inf \left\{\overline{\operatorname{dim}}_{b} A: A \in \mathscr{B}(X), \mu(A)>0\right\} \geq \mathrm{sl}-\overline{\operatorname{dim}}_{b} X .
$$

Proof. Fix $0<\lambda<$ sl- $\overline{\operatorname{dim}}_{b} X$ and $\kappa>0$. Given $x \in X$ and $r, \varepsilon>0$, denote by $M(x, r ; \varepsilon)$ the greatest possible number of disjoint closed balls of radius $\varepsilon$ that may be found with centers in the ball $B(x, r)$. Since $\overline{\operatorname{dim}}_{b} B(x, r)>\lambda$, there is $\varepsilon \in(0,1)$ such that

$$
M(x, r ; \varepsilon) \geq \varepsilon^{-\lambda} .
$$

For every $x \in X$ and $r>0$, we fix an $\varepsilon=\varepsilon(x, r)$ satisfying the last inequality. In the ball $B(x, r)$, we choose the points $y_{1}, \ldots, y_{M}$, where $M=M(x, r ; \varepsilon)$, such that the balls with centers at $y_{i}$ for $i \in\{1, \ldots, M\}$ and radius $\varepsilon$ are disjoint.

Define

$$
\mu_{x, r}=\frac{1}{M}\left(\delta_{y_{1}}+\cdots+\delta_{y_{M}}\right)
$$

Clearly $\mu_{x, r} \in \mathcal{M}_{1}(X)$. As previously, denote by $\mathscr{F}$ the family of all finite subsets of $X$.

For given $F \in \mathscr{F}$ and $r>0$, we denote by $k(F)$ the number of elements of $F$ and we define

$$
\begin{gathered}
\mu_{F, r}=\frac{1}{k(F)} \sum_{x \in F} \mu_{x, r}, \\
\alpha(F, r)=\frac{\kappa}{6} \min \{\varepsilon(x, r): x \in F\}, \\
\mathscr{D}(F, r)=\left\{\mu \in M_{1}(X): d_{\mathrm{FM}}\left(\mu, \mu_{F, r}\right)<\alpha(F, r)\right\} .
\end{gathered}
$$

Now, for $m \in \mathbb{N}$, define

$$
\mathscr{G}_{m}=\bigcup_{n=m}^{\infty} \mathscr{D}\left(\frac{1}{n}\right), \quad \text { where } \mathscr{D}\left(\frac{1}{n}\right)=\bigcup_{F \in \mathscr{F}} \mathscr{D}\left(F, \frac{1}{n}\right) .
$$

It is easy to see that the set $\mathscr{G}_{m}$ is open and dense in $\mathcal{M}_{1}(X)$. Recall that $\mu_{F, r}$ and, consequently, $\mathscr{D}(F, 1 / n)$ and $\mathscr{G}_{m}$ are constructed for fixed $\lambda \in\left(0\right.$, sl $\left.-\overline{\operatorname{dim}}_{b} X\right)$ and $\kappa>0$. Now, for 
such $\lambda$ and $\kappa$, we define

$$
\mathscr{G}(\lambda, \kappa)=\bigcap_{m=1}^{\infty} \mathscr{G}_{m}
$$

Clearly the set $\mathscr{G}(\lambda, \kappa)$ is residual in the space $M_{1}(X)$.

It can be proved that for arbitrary $\mu \in \mathscr{G}(\lambda, \kappa)$ we have

$$
\overline{\operatorname{dim}}_{b} A \geq \lambda \quad \text { for every } A \in \mathscr{B}(X) \text { with } \mu(A) \geq \kappa .
$$

Now, let $\left(\lambda_{n}\right)$ be a strictly increasing sequence of positive numbers convergent to sl- $\overline{\operatorname{dim}}_{b} X$ and let $\left(\kappa_{n}\right)$ be a decreasing sequence of positive numbers convergent to 0 . Clearly the set

$$
\mathscr{G}=\bigcap_{n=1}^{\infty} \mathscr{G}\left(\lambda_{n}, \kappa_{n}\right)
$$

is residual in $M_{1}(X)$. Let $\mu \in \mathscr{G}$. Since for arbitrary $n \in \mathbb{N}$ we have $\mu \in \mathscr{G}\left(\lambda_{n}, \kappa_{n}\right)$, then

$$
\overline{\operatorname{dim}}_{b} A \geq \lambda_{n} \quad \text { for every } A \in \mathscr{B}(X) \text { with } \mu(A) \geq \kappa_{n},
$$

whence the statement of Theorem 4.2 follows immediately.

As an immediate consequence of Theorem 4.2, we have the following corollary.

Corollary 4.3. For a typical measure $\mu \in M_{1}(X)$,

$$
\overline{\operatorname{dim}}_{b} \mu \geq \operatorname{sl}-\overline{\operatorname{dim}}_{b} X .
$$

Theorem 4.4. Assume that $(X, \rho)$ is a complete separable metric space. Then for a typical measure $\mu \in M_{1}(X)$,

$$
\operatorname{supp} \mu=X
$$

Proof. Let $\left(x_{n}\right)_{n \in \mathbb{N}}$ be a sequence of elements of $X$ such that for each $n_{0}$ the subsequence $\left(x_{n}\right)_{n \geq n_{0}}$ is dense in $X$. Let $\mathbf{P}$ be the set of all real sequences $\mathbf{p}=\left(p_{n}\right)_{n \in \mathbb{N}}$ such that $p_{n}>0$ for all $n \in \mathbb{N}$ and $\sum_{n=1}^{\infty} p_{n}=1$.

For $\mathbf{p} \in \mathbf{P}$ and $n \in \mathbb{N}$, we define

$$
\begin{gathered}
\mu_{\mathbf{p}}=\sum_{n=1}^{\infty} p_{n} \delta_{x_{n}}, \quad \alpha(\mathbf{p}, n)=\frac{1}{n} \min _{1 \leq i \leq n} p_{i}, \\
\mathscr{D}_{n}(\mathbf{p})=\left\{\mu \in M_{1}(X): d_{\mathrm{FM}}\left(\mu, \mu_{\mathbf{p}}\right)<\alpha(\mathbf{p}, n)\right\} .
\end{gathered}
$$

Let

$$
\mathscr{D}=\bigcap_{n=1}^{\infty} \mathscr{D}_{n}, \quad \text { where } \mathscr{D}_{n}=\bigcup_{\mathbf{p} \in \mathbf{P}} \mathscr{D}_{n}(\mathbf{p}) \text {. }
$$


Since for each $n \in \mathbb{N}$ the set $\mathscr{D}_{n}$ is dense and open in the space $\mathcal{M}_{1}(X)$, the set $\mathscr{D}$ is residual in $M_{1}(X)$. To complete the proof it suffices to show that $\operatorname{supp} \mu=X$ for every $\mu \in \mathscr{D}$.

From Theorem 4.4, follows immediately the following corollary.

Corollary 4.5. Let $(X, \rho)$ be a complete separable metric space. Then for a typical measure $\mu$ in $M_{1}(X)$,

$$
\underline{\operatorname{dim}}_{b} A=\underline{\operatorname{dim}}_{b} X, \quad \overline{\operatorname{dim}}_{b} A=\overline{\operatorname{dim}}_{b} X
$$

for every $A \in \mathscr{B}(X)$ such that $\mu(A)=1$.

\section{Correlation dimension of measures}

Given $\mu \in M_{1}(X)$, we define the upper and lower correlation dimension of $\mu$ by the formulae

$$
\begin{aligned}
& \overline{\operatorname{dim}}_{c} \mu=\limsup _{r \rightarrow 0^{+}} \frac{1}{\log r} \log \int_{X} \mu(B(x, r)) d \mu(x), \\
& \underline{\operatorname{dim}}_{c} \mu=\liminf _{r \rightarrow 0^{+}} \frac{1}{\log r} \log \int_{X} \mu(B(x, r)) d \mu(x) .
\end{aligned}
$$

Note that if $\mu(\{x\})>0$ for some $x \in X$, then $\overline{\operatorname{dim}}_{c} \mu=\underline{\operatorname{dim}}_{c} \mu=0$. The correlation dimension introduced by Procaccia et al. [30] (see also [29]) is frequently used in the theory of dynamical systems.

Theorem 5.1. For a typical measure $\mu \in M_{1}(X)$,

$$
\underline{\operatorname{dim}}_{c} \mu=0 .
$$

Proof. Let $\left(\varepsilon_{n}\right)$ and $\left(\delta_{n}\right)$ be sequences of positive numbers convergent to zero. For $n \in \mathbb{N}$, we set

$$
\begin{gathered}
\mathcal{N}_{n}=\left\{\nu \in \mathcal{M}_{1}(X): \nu\left(\left\{x_{0}\right\}\right) \geq \varepsilon_{n} \text { for some } x_{0} \in X\right\} \\
\mathscr{G}_{n}=\bigcup_{\nu \in \mathcal{N}_{n}}\left\{\mu \in \mathcal{M}_{1}(X): d_{\mathrm{FM}}(\mu, \nu)<\delta_{n}\right\} .
\end{gathered}
$$

Let

$$
\mathscr{G}=\bigcap_{m=1}^{\infty} \bigcup_{n=m}^{\infty} \mathscr{G}_{n} .
$$

Since $\mathscr{G}$ is a residual subset of $\mathcal{M}_{1}(X)$ to complete the proof, it suffices to show that $\underline{\operatorname{dim}}_{c} \mu=0$ for every $\mu \in \mathscr{G}$.

Theorem 5.2. For a typical measure $\mu \in M_{1}(X)$,

$$
\text { sl- } \underline{\operatorname{dim}}_{b} X \leq \overline{\operatorname{dim}}_{c} \mu \leq \mathrm{sl}-\overline{\operatorname{dim}}_{b} X .
$$


Sketch of the proof. One can prove that for every $n \in \mathbb{N}$ the sets

$$
\begin{aligned}
\mathscr{G}_{\alpha-1 / n} & =\left\{\mu \in \mathcal{M}_{1}(X): \overline{\operatorname{dim}}_{c} \mu \geq \alpha-\frac{1}{n}\right\}, \\
\varphi^{\beta+1 / n} & =\left\{\mu \in \mathcal{M}_{1}(X): \overline{\operatorname{dim}}_{c} \mu<\beta+\frac{1}{n}\right\},
\end{aligned}
$$

where $\alpha=s l-\underline{\operatorname{dim}}_{b} X$ and $\beta=$ sl- $\overline{\operatorname{dim}}_{b} X$, are residual in $M_{1}(X)$. Let

$$
\varphi_{\alpha}^{\beta}=\bigcap_{n=1}^{\infty}\left(\varphi_{\alpha-1 / n} \cap \varphi^{\beta+1 / n}\right) .
$$

Since the set $\varphi_{\alpha}^{\beta}$ is residual in $\mu_{1}(X)$ and for every $\mu \in \varphi_{\alpha}^{\beta}$ the inequalities (5.5) hold, the proof is complete.

Note that the estimation in Theorem 5.2 cannot be improved. In fact, we can construct a Cantor-like set $C$ with $\operatorname{dim}_{b} C=0, \overline{\operatorname{dim}}_{b} C=1$ such that the set $\left\{\mu \in M_{1}(C)\right.$ : $\left.\overline{\operatorname{dim}}_{c} \mu=1\right\}$ is residual in $\mu_{1}(C)$. On the other hand, we can construct a set $X \subset \mathbb{R}$ such that $\operatorname{dim}_{b} B(x, r)=1$ for all $x \in X$ and $r>0$ but $\overline{\operatorname{dim}}_{c} \mu=0$ for $\mu$ from some open and dense subset $\mathscr{G}$ of $\mathcal{M}_{1}(X)$ (see [23]).

\section{Concentration dimension of measures}

Given $\mu \in \mathcal{M}_{1}(X)$, we define the lower and upper concentration dimension of $\mu$ by the formulae

$$
\begin{aligned}
& \underline{\operatorname{dim}}_{L} \mu=\liminf _{r \rightarrow 0^{+}} \frac{\log \Phi_{\mu}(r)}{\log r}, \\
& \overline{\operatorname{dim}}_{L} \mu=\limsup _{r \rightarrow 0^{+}} \frac{\log \Phi_{\mu}(r)}{\log r},
\end{aligned}
$$

where

$$
\Phi_{\mu}(r)=\sup _{x \in X} \mu(B(x, r))
$$

is a well-known Lévy concentration function. Such function was successfully used to study the convergence of sequences of random variables. For the properties and other applications of concentration function, see [13].

Clearly, lower and upper concentration dimension are well defined and nonnegative, however, they can be infinite. If $\overline{\operatorname{dim}}_{L} \mu=\underline{\operatorname{dim}}_{L} \mu$, this common value is called concentration dimension.

The concentration dimension, introduced in [18], is strongly related with the Hausdorff dimension. It is easy to prove that the Hausdorff dimension of measure $\mu$ is greater than or equal to the lower concentration dimension of $\mu$. Moreover, if $A$ is a nonempty compact set, then the following variational principle holds:

$$
\operatorname{dim}_{H} A=\sup \left\{\underline{\operatorname{dim}}_{L} \mu: \mu \in \mathcal{M}_{1}(X) \text { with supp } \mu \subset A\right\} .
$$


It is also interesting that concentration dimension is greater than or equal to the topological dimension (see [26]). In particular, if $X$ is a Polish space in [27], it is proved that

$$
\operatorname{dim}_{T}=\inf \left\{\operatorname{dim}_{L} X^{\prime}: X^{\prime} \text { is homeomorphic to } X\right\}
$$

where $\operatorname{dim}_{T}$ stands for the topological dimension of $X$ and $\operatorname{dim}_{L} X$ for the concentration dimension of $X$ defined by formula

$$
\operatorname{dim}_{L} X=\sup \left\{\underline{\operatorname{dim}}_{L} \mu: \mu \in M_{1}(X)\right\} .
$$

Note that the concentration dimension seems to be more easy to be calculated. For example, in [18] the concentration dimension was calculated for invariant measures corresponding to some iterated function systems and invariant measures corresponding to a partial differential equation.

A subset $K$ of $X$ is called quasi-self-similar from below if there exist $a>0$ and $r_{0}>0$ such that for every ball $B(x, r)$ with center at $x \in K$ and radius $r \in\left(0, r_{0}\right)$ there is a mapping $\varphi: K \rightarrow K \cap B(x, r)$ such that

$$
\operatorname{ar} \rho(x, y) \leq \rho(\varphi(x), \varphi(y)) \quad \forall x, y \in K
$$

These sets are a natural generalization of self-similar sets which appears in the theory of iterated function systems. Namely, let $S_{i}: X \rightarrow X, i=1, \ldots, N$, be strictly contracting similarity transformations. It is well known (see [14]) that there is a unique compact set $K$ such that $K=\bigcup_{k=1}^{N} S_{i}(K)$. Such set, called self-similar fractal, a obviously satisfies condition (6.6). In fact, in this case, the function $\varphi$ satisfies also the quasi-self-similarity condition from above, and the corresponding set $K$ is called quasi-self-similar. For details see [4, Example 2]. Note that quasi-self-similar sets appear in the theory of dynamical systems and were intensively studied by several authors (see $[5,19,32])$. K.

Let $M_{1}(K)$ denote the family of all probability Borel measures on $X$ such that $\operatorname{supp} \mu \subset$

Theorem 6.1. Let $K$ be a compact quasi-self-similar from below subset of $X$. Then for a typical measure $\mu \in M_{1}(K)$,

$$
\underline{\operatorname{dim}}_{L} \mu=0
$$

Proof. Let

$$
\mathcal{N}=\left\{\mu \in M_{1}(K): \underline{\operatorname{dim}}_{L} \mu=0\right\} .
$$

From the equality $\operatorname{dim}_{L} \delta_{x}=0$ and the fact that linear combinations of point Dirac measures are dense in the space $M_{1}(K)$, it follows that $\mathcal{N}$ is dense in $M_{1}(K)$.

Let $v \in \mathcal{N}$ and $n \in \mathbb{N}$. From condition $\underline{\operatorname{dim}}_{L} v=0$, it follows that there exists $r_{v, n} \in$ $(0,1 / n)$ such that $\Phi_{\nu}\left(r_{v, n}\right) \geq 2\left(r_{v, n}\right)^{1 / n}$. For every $\nu \in \mathcal{N}$ and $n \in \mathbb{N}$, we choose such $r_{v, n}$ and we define

$$
\mathscr{D}_{n}(\nu)=\left\{\mu \in \mathcal{M}_{1}(K): d_{\mathrm{FM}}(\mu, \nu)<\left(r_{\nu, n}\right)^{(n+1) / n}\right\}
$$


Now, we define

$$
D=\bigcap_{n=1}^{\infty} \mathscr{D}_{n}, \quad \text { where } \mathscr{D}_{n}=\bigcup_{\nu \in \mathcal{N}} \mathscr{D}_{n}(\nu) .
$$

Since $\mathscr{D}_{n}, n \in \mathbb{N}$, are open and dense in $\mathcal{M}_{1}(K)$, the set $\mathscr{D}$ is residual. To complete the proof it suffices to verify that $\underline{\operatorname{dim}}_{L} \mu=0$ for every $\mu \in \mathscr{D}$.

Theorem 6.2. Let $K$ be a compact quasi-self-similar from below subset of $X$. Then for a typical measure $\mu \in M_{1}(K)$,

$$
\overline{\operatorname{dim}}_{L} \mu=\operatorname{dim}_{H} K .
$$

Proof. Set $d=\operatorname{dim}_{H} K$. For $n \in \mathbb{N}$, define

$$
\mathcal{N}_{n}=\left\{\mu \in M_{1}(K): \overline{\operatorname{dim}}_{L} \mu>d-\frac{1}{n}\right\} .
$$

Using the quasi-self-similarity assumption on $K$ and the fact that linear combinations of point Dirac measures are dense in $\mathcal{M}_{1}(K)$, one can prove that the set $\mathcal{N}_{n}$ is dense in $M_{1}(K)$.

Now, for $v \in \mathcal{N}_{n}$, we define

$$
\mathscr{G}_{n}(\nu)=\left\{\mu \in \mathcal{M}_{1}(K): d_{F H}(\mu, \nu)<\left(r_{v, n}\right)^{d+(n-1) / n}\right\},
$$

where $r_{v, n}<1 / n$ and such that $\Phi_{\nu}\left(2 r_{\nu, n}\right) \leq\left(r_{\nu, n}\right)^{d-1 / n}$.

Define

$$
\mathscr{G}=\bigcap_{n=1}^{\infty} \mathscr{G}_{n}, \quad \text { where } \mathscr{G}_{n}=\bigcup_{\nu \in \mathcal{N}_{n}} \mathscr{G}_{n}(\nu) .
$$

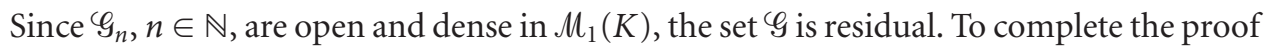
it suffices to show that $\overline{\operatorname{dim}}_{L} \mu=d$ for every $\mu \in \mathscr{G}$.

\section{Local dimension of measures}

Given $\mu \in M_{1}(X)$ and $x \in X$, we define the lower and upper local dimension of $\mu$ at point $x$ by the formulae

$$
\begin{aligned}
& 1-\underline{\operatorname{dim}} \mu(x)=\liminf _{r \rightarrow 0} Q_{\mu}(x, r), \\
& 1-\overline{\operatorname{dim}} \mu(x)=\limsup _{r \rightarrow 0} Q_{\mu}(x, r),
\end{aligned}
$$

where

$$
Q_{\mu}(x, r)=\frac{\log \mu(B(x, r))}{\log r} .
$$

Note that the local dimension does not change if we replace the closed ball $B(x, r)$ by the open ball $B^{o}(x, r)$. From Alexandrov's theorem, it follows immediately that given $x \in X$ and $r>0$, the function $M_{1}(X) \ni \mu \mapsto Q_{\mu}(x, r) \in \mathbb{R}_{+}$is lower semicontinuous in the case of closed balls and upper semicontinuous in the case of open balls. 
Theorem 7.1. Let $X$ be a Polish space. For a typical measure $\mu \in M_{1}(X)$, there is a residual subset $A_{\mu}$ of $X$ such that $1-\underline{\operatorname{dim}} \mu(x)=0$ for every $x \in A_{\mu}$. If $X$ has no isolated points, then the set $A_{\mu}$ can be chosen in such a way that $1-\overline{\operatorname{dim}} \mu(x)=\infty$ for every $x \in A_{\mu}$.

Proof. Consider first the case of upper local dimension. For $x \in X, n \in \mathbb{N}$, and $a>0$, we define

$$
\mathscr{G}_{x, 1 / n, a}=\left\{\mu \in M_{1}(X): \sup _{0<r<1 / n} Q_{\mu}(x, r)>a\right\} .
$$

From the lower semicontinuity of function $\mu \mapsto Q_{\mu}(x, r)$, it follows that the set $\mathscr{G}_{x, 1 / n, a}$ is open.

We claim that the set $\mathscr{G}_{x, 1 / n, a}$ is also dense in $\mathcal{M}_{1}(X)$. Indeed, let $\mu \in \mathcal{M}_{1}(X) \backslash \mathscr{G}_{x, 1 / n, a}$ and $\varepsilon>0$. Without loss of generality, we can assume that $\mu(\{x\})=0$ (if $\mu(\{x\})>0$ and $X$ has no isolated points, we can find a measure $\tilde{\mu} \in M_{1}(X)$ such that $\left.d_{\mathrm{FM}}(\mu, \tilde{\mu})<\varepsilon / 2\right)$. Choose $s>a$ and $r<1 / n$ such that $\mu(B(x, r))<\varepsilon / 2$. Now we define $\mu_{\varepsilon} \in M_{1}(X)$ as follows:

$$
\mu_{\varepsilon}(B(x, r))=r^{s}, \quad \mu_{\varepsilon}(A)=\frac{1-r^{s}}{(1-\mu(B(x, r))) \mu(A)} \quad \text { for } A \subset X \backslash B(x, r) .
$$

Simple calculation shows that $h_{\mathrm{FM}}\left(\mu, \mu_{\varepsilon}\right)<\varepsilon$. Since $\mu_{\varepsilon} \in \mathscr{G}_{x, 1 / n, a}$, the claim is proved.

Let $\left(x_{i}\right)$ be a sequence dense in $X$. Define

$$
\mathscr{G}_{a}=\bigcap_{n=1}^{\infty} \bigcap_{i=1}^{\infty}\left\{\mu \in M_{1}(X): \sup _{0<r<1 / n} Q_{\mu}\left(x_{i}, r\right)>a\right\} .
$$

Clearly $\mathscr{G}_{a}$ is a residual subset of $\mathcal{M}_{1}(X)$.

Now, given $\mu \in \mathscr{G}_{a}$ and $n \in \mathbb{N}$, we define

$$
A_{\mu, 1 / n, a}=\left\{x \in X: \sup _{0<r<1 / n} Q_{\mu}(x, r)>a\right\} .
$$

Since $A_{\mu, 1 / n, a}$ is a dense open subset of $X$, the set

$$
A_{\mu, a}=\bigcap_{n=1}^{\infty} A_{\mu, 1 / n, a}
$$

is residual in $X$. It is easy to verify that for $\mu \in \mathscr{G}_{a}$ we have $1-\operatorname{dim} \mu(x) \geq a$ for every $x \in$ $A_{\mu, a}$. Now set

$$
\mathscr{G}=\bigcap_{m=1}^{\infty} \mathscr{G}_{m}
$$

and for $\mu \in \mathscr{G}$ define

$$
A_{\mu}=\bigcap_{m=1}^{\infty} A_{\mu, m}
$$

Observe that $1-\overline{\operatorname{dim}} \mu(x)=\infty$ for every $x \in A_{\mu}$. Since $\mathscr{G}$ is residual in $\mu_{1}(X)$ and $A_{\mu}$ is residual in $X$, the proof for the case of upper local dimension is complete. 
For the lower local dimension, the argument is similar. Using the open balls, we can show that the set

$$
\mathscr{D}_{a}=\bigcap_{n=1}^{\infty} \bigcap_{i=1}^{\infty}\left\{\mu \in M_{1}(X): \sup _{0<r<1 / n} Q_{\mu}(x, r)<a\right\}
$$

is residual in $M_{1}(X)$ for every $a>0$. Moreover, we can show that for $\mu \in \mathscr{D}_{a}$ the set

$$
C_{\mu, a}=\bigcap_{n=1}^{\infty}\left\{x \in X: \sup _{0<r<1 / n} Q_{\mu}(x, r)<a\right\}
$$

is residual in $X$. To complete the proof, it suffices to take

$$
\mathscr{D}=\bigcap_{m=1}^{\infty} \mathscr{D}_{1 / m}
$$

and for $\mu \in \mathscr{D}$ the set

$$
C_{\mu}=\bigcap_{m=1}^{\infty} C_{\mu, 1 / m}
$$

For further properties of local dimensions, see $[8,11]$.

\section{References}

[1] W. Bartoszek, On the residuality of mixing by convolutions probabilities, Israel J. Math. 80 (1992), no. 1-2, 183-193.

[2] J. R. Choksi and V. S. Prasad, Approximation and Baire category theorems in ergodic theory, Measure Theory and Its Applications (Sherbrooke, Que., 1982), Lecture Notes in Math., vol. 1033, Springer, Berlin, 1983, pp. 94-113.

[3] R. M. Dudley, Probabilities and Metrics, Lecture Notes Series, no. 45, Matematisk Institut, Aarhus Universitet, Aarhus, 1976.

[4] K. J. Falconer, Dimensions and measures of quasi self-similar sets, Proc. Amer. Math. Soc. 106 (1989), no. 2, 543-554.

[5] _ Techniques in Fractal Geometry, John Wiley \& Sons, Chichester, 1997.

[6] D. Feng and J. Wu, Category and dimension of compact subsets of $\mathbf{R}^{\mathbf{n}}$, Chinese Sci. Bull. 42 (1997), no. 20, 1680-1683.

[7] R. Fortet and E. Mourier, Convergence de la répartition empirique vers la répartition théorique, Ann. Sci. Ecole Norm. Sup. (3) 70 (1953), 267-285 (French).

[8] J. Genyuk, A typical measure typically has no local dimension, Real Anal. Exchange 23 (1997/1998), no. 2, 525-537.

[9] P. M. Gruber, Results of Baire category type in convexity, Discrete Geometry and Convexity (New York, 1982), Ann. New York Academic Science, vol. 440, New York Acad. Sci., New York, 1985, pp. 163-169.

[10] Dimension and structure of typical compact sets, continua and curves, Monatsh. Math. 108 (1989), no. 2-3, 149-164.

[11] H. Haase, A survey on the dimension of measures, Topology, Measures, and Fractals (Warnemünde, 1991), Math. Res., vol. 66, Akademie Verlag, Berlin, 1992, pp. 66-75.

[12] J. Hawkes, Hausdorff measure, entropy, and the independence of small sets, Proc. London Math. Soc. (3) 28 (1974), 700-724. 
[13] W. Hengartner and R. Theodorescu, Concentration Functions, Probability and Mathematical Statistics, no. 20, Academic Press, New York, 1973.

[14] J. E. Hutchinson, Fractals and self-similarity, Indiana Univ. Math. J. 30 (1981), no. 5, 713-747.

[15] A. Iwanik, Baire category of mixing for stochastic operators, Measure Theory Conference (Oberwolfach , 1990), Rend. Circ. Mat. Palermo (2) Suppl. no. 28, 1992, pp. 201-217.

[16] A. Lasota and J. Myjak, Generic properties of stochastic semigroups, Bull. Polish Acad. Sci. Math. 40 (1992), no. 4, 283-292.

[17] - Generic properties of fractal measures, Bull. Polish Acad. Sci. Math. 42 (1994), no. 4, 283-296.

[18] _ On a dimension of measures, Bull. Polish Acad. Sci. Math. 50 (2002), no. 2, 221-235.

[19] J. McLaughlin, A note on Hausdorff measures of quasi-self-similar sets, Proc. Amer. Math. Soc. 100 (1987), no. 1, 183-186.

[20] J. Myjak and R. Rudnicki, Box and packing dimensions of typical compact sets, Monatsh. Math. 131 (2000), no. 3, 223-226.

[21] - On the typical structure of compact sets, Arch. Math. (Basel) 76 (2001), no. 2, 119-126.

[22] - On the box dimension of typical measures, Monatsh. Math. 136 (2002), no. 2, 143-150.

[23] Typical properties of correlation dimension, Real Anal. Exchange 28 (2002/2003), no. 2, 269-278.

[24] J. Myjak and T. Szarek, Generic properties of Markov operators, Rend. Circ. Mat. Palermo (2) Suppl. (2002), no. 70, part II, 191-200.

[25] - Szpilrajn type theorem for concentration dimension, Fund. Math. 172 (2002), no. 1, $19-25$.

[26] Some generic properties of concentration dimension of measure, Boll. Unione Mat. Ital. Sez. B Artic. Ric. Mat. (8) 6 (2003), no. 1, 211-219.

[27] J. Myjak, T. Szarek, and M. Ślęczka, Szpilrajn-Marczewski type theorem for concentration dimension on Polish spaces, Canad. J. Math. (2005).

[28] A. J. Ostaszewski, Families of compact sets and their universals, Mathematika 21 (1974), 116127.

[29] Ya. B. Pesin, On rigorous mathematical definitions of correlation dimension and generalized spectrum for dimensions, J. Statist. Phys. 71 (1993), no. 3-4, 529-547.

[30] L. Procaccia, P. Grassberger, and H. G. E. Hentschel, On the characterization of chaotic motions, Dynamical Systems and Chaos (Sitges/Barcelona, 1982), Lecture Notes in Phys., vol. 179, 1983, pp. 212-222.

[31] R. Rębowski, Most Markov operators on $C(X)$ are quasicompact and uniquely ergodic, Colloq. Math. 52 (1987), no. 2, 277-280.

[32] D. Sullivan, Seminar on Conformal and Hyperbolic Geometry, Lecture Notes, Institute des Hautes Etudes Scientifiques, Bures-sur-Yvette, 1982.

[33] M. Tyran-Kamińska, Generic properties of iterated function systems with place dependent probabilities, Univ. Iagel. Acta Math. 35 (1997), 213-223.

[34] T. Zamfirescu, Using Baire categories in geometry, Rend. Sem. Mat. Univ. Politec. Torino 43 (1985), no. 1, 67-88.

[35] _ Porosity in convexity, Real Anal. Exchange 15 (1989/90), no. 2, 424-436.

[36] _ Baire categories in convexity, Atti Sem. Mat. Fis. Univ. Modena 39 (1991), no. 1, 139164.

Józef Myjak: Dipartimento di Matematica Pura ed Applicata, Università dell'Aquila, via Vetoio, 67010 l'Aquila, Italy

E-mail address: myjak@univaq.it

Current address: Wydzial Matematyki Stosowanej, Akademia Górniczo-Hutnicza, Al. Mickiewicza 30, 30059 Kraków, Poland 


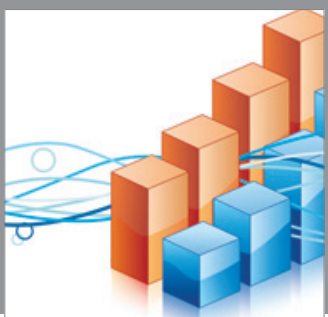

Advances in

Operations Research

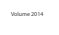

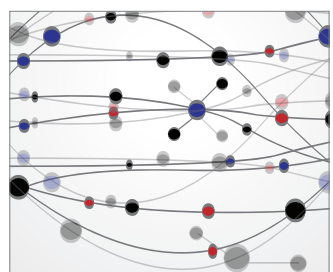

\section{The Scientific} World Journal
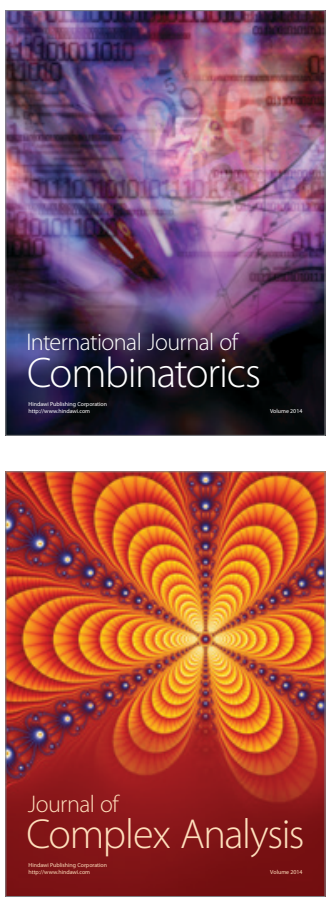

International Journal of

Mathematics and

Mathematical

Sciences
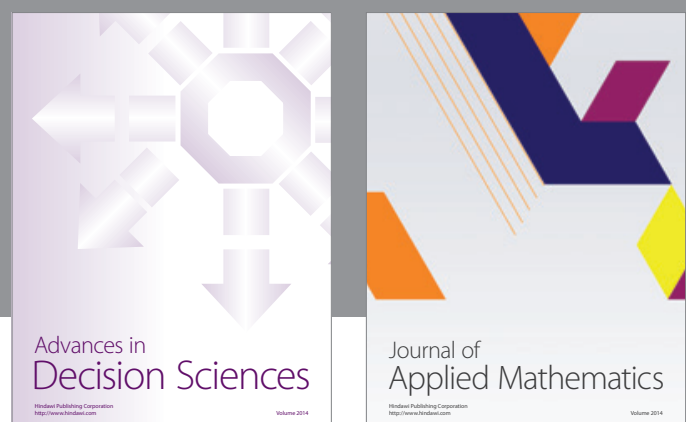

Journal of

Applied Mathematics
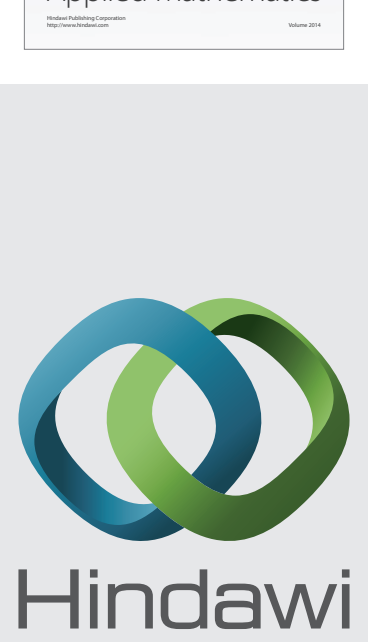

Submit your manuscripts at http://www.hindawi.com
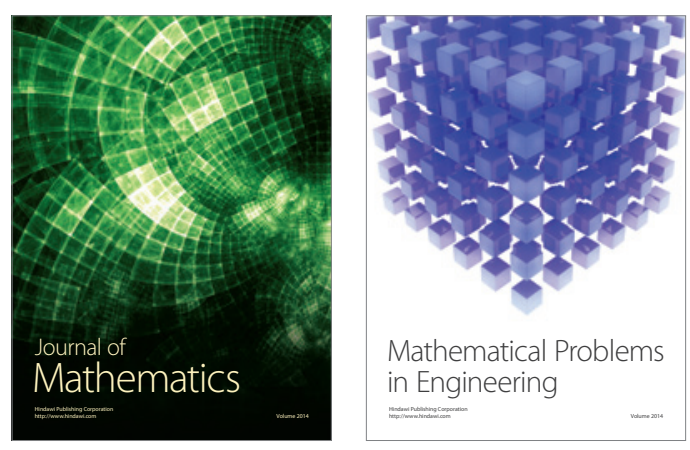

Mathematical Problems in Engineering
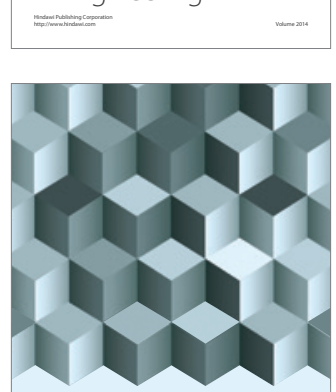

Journal of

Function Spaces
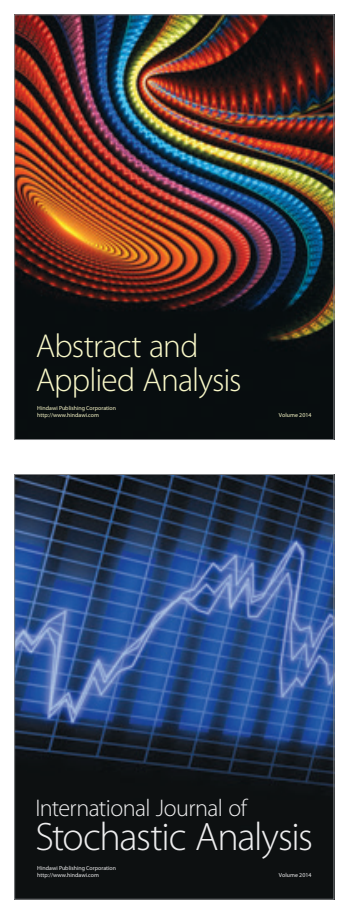

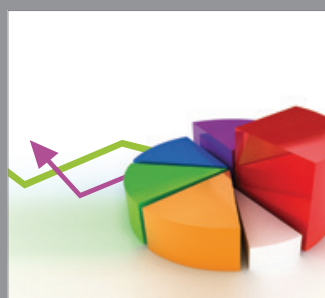

ournal of

Probability and Statistics

Promensencen
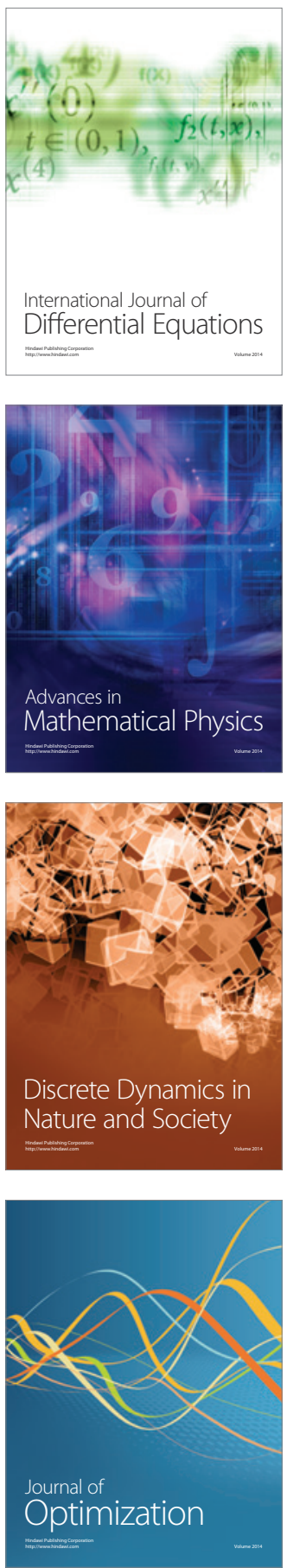